\title{
Screening for candidate genes involved in the production of mouse subventricular zone proliferative cells and an estimation of their changes in evolutionary pressure during primate evolution
}

\author{
Hidenori Tabata ${ }^{1,2 *}{ }^{+}$, Tsuyoshi Hachiya $^{3,4+}$, Koh-ichi Nagata ${ }^{2}$, Yasubumi Sakakibara $^{3}$ and \\ Kazunori Nakajima ${ }^{1 *}$
}

1 Department of Anatomy, School of Medicine, Keio University, Tokyo, Japan

2 Department of Molecular Neurobiology, Institute for Developmental Research, Aichi Human Service Center, Kasugai, Japan

${ }^{3}$ Department of Biosciences and Informatics, Keio University, Yokohama, Japan

${ }^{4}$ Iwate Medical Megabank Organization, Iwate Medical University, Shiwa, Japan

\section{Edited by:}

Eric Lewitus, Max Planck Institute for Molecular Cell Biology and Genetics, Germany

\section{Reviewed by:}

Veronica M. Cerdeño, University of California at Davis, USA

Victor Borrell, Consejo Superior de

Investigaciones Científicas, Spain

\section{*Correspondence:}

Hidenori Tabata, Department of

Molecular Neurobiology, Institute for Developmental Research, 713-8

Kamiya, Kasugai, Aichi 480-0392,

Japan

e-mail: tabata@inst-hsc.jp;

Kazunori Nakajima, Department of Anatomy, School of Medicine,

Keio University, 35 Shinanomachi, Shinjuku, Tokyo 160-8582, Japan

e-mail: kazunori@z6.keio.jp

tThese authors have contributed equally to this work.
During neocortical development, excitatory neurons are produced from apical progenitors in the ventricular zone (VZ) or from dividing cells in the subventricular zone (SVZ). We previously reported that the direct progenies of $V Z$ cells in mice slowly exit the $V Z$ and accumulate just above the VZ (lower SVZ) as multipolar migrating neurons, whereas subsequently dividing cells in the SVZ exit the VZ earlier than the former and become widely distributed in the SVZ. These two populations are named the slowly exiting population (SEP) and the rapidly exiting population (REP), respectively. In mice, REP cells include basal progenitors as the major population and are characterized by a long ascending process; their morphology resembles that of basal radial glial cells (bRGs), which have been observed in the inner and outer SVZ in primates. The dramatic increase in the number of bRGs in primates, especially in humans, is thought to underlie the acquisition of a huge cortex during evolution. We previously reported that the REP/SEP production rate in the lateral cortical VZ is higher than that in the dorsomedial VZ in mice. To search for molecules responsible for the higher REP production in the lateral cortical VZ, we conducted microarray analyses and identified genes that were differentially expressed between the lateral and medial VZs in mice. These genes were considered to be among the candidates responsible for the regulation of the REP/SEP production rate. To investigate the selection pressures during primate evolution on these candidate genes, we estimated the synonymous vs. non-synonymous base substitution rates. As a result, the negative selection pressures on the Megf11, Dmrt3, and Cntn3 genes were found to be significantly weaker in primates than in non-primates, while those on Jag1, Ntrk2, and Pmp22 were stronger. Candidate molecules responsible for primate cortical expansion through an increase in bRGs may be included among these genes.

Keywords: neural stem cells, human evolution, notch signaling, selection bias, subventricular zone (SVZ)

\section{INTRODUCTION}

During evolution, humans have acquired a huge cerebral cortex that is thought to be the base of our higher mental activities. The cortical neurons in mammals are generated from the ventricular zone (VZ) lining the lateral ventricles or the adjacent subventricular zone (SVZ) during the embryonic or fetal stages. In murine embryos, neural stem cells exist in the VZ and are called radial glia (RG) (Noctor et al., 2001). These cells extend a long, ascending (basal) process, which is also called a radial fiber, toward the pial surface while a short thick apical process reaches the ventricular surface. During the neurogenic cell divisions, RG undergo asymmetric cell divisions to produce one RG and one neuron. In many instances of this asymmetric cell division, the daughter cells that have inherited the ascending process become RG, especially during mid or late cortical development (Konno et al., 2007). RG also produce proliferative cells in the SVZ, and most of the proliferative cells in the murine SVZ undergo symmetric cell division to produce two neurons (Noctor et al., 2004). This cell division is terminal, and this type of progenitor cells in the SVZ is called the basal progenitor (BP). They are proliferative but do not have self-renewing ability and contribute to temporal amplification of neuron production. In that sense, they are also called intermediate progenitor cells (IPCs).

We previously observed the initial phase of the migration of cells exiting the VZ during late cortical plate development in mice (embryonic day [E] 14.5 16) and described two distinct populations (Tabata et al., 2009). One population finishes their final cell division in the VZ, remains there for more than $10 \mathrm{~h}$, and then exits from the VZ and accumulates in the lower SVZ, where the cells exhibit a multipolar cell morphology (Tabata and 
Nakajima, 2003). We named this region the multipolar cell accumulation zone (MAZ) to avoid confusion with the term SVZ, which is defined as the region containing proliferative cells not attached to the ventricular surface and not showing interkinetic nuclear movement during the mitotic cycle (Boulder Committee, 1970). After remaining in the MAZ for about $24 \mathrm{~h}$, the multipolar neurons then extend an axon tangentially and resume their migration using a locomotion mode in which the cells assume a bipolar morphology with a thick leading process and a thin trailing process and migrate using the radial fiber as a scaffold. The other population exits from the VZ faster than the former population and uses a somal translocation mode in which cells with a long ascending process retract this process to translocate the cell body while the tip of the ascending process remains attached to the brain surface; these cells divide in the intermediate zone (IZ) or SVZ. As these cell populations differ from each other in the timing of their exit from the VZ, we named them the slowly exiting population (SEP) and the rapidly exiting population (REP), respectively. Because the SEP includes all the cells that have completed cell division while in the VZ, it includes the daughter cells of short neural progenitors (SNPs), which have a short ascending fiber in the VZ and produce two neurons as the apical intermediate progenitors (Gal et al., 2006). Among the REP cells, 7.6\% expressed Olig2, a glial progenitor marker, and $65 \%$ expressed NeuroD, a neuronal marker, at $36 \mathrm{~h}$ after the introduction of a GFP expression vector using in utero electroporation (Tabata et al., 2009). These results indicated that the REP includes some glial progenitors, while the major population is neurogenic.

In primate fetal cortices, including those of humans, the SVZ becomes enormously thick as the development proceeds and is divided into two histologically distinct regions, the outer SVZ (OSVZ) and the inner SVZ (ISVZ) (Smart et al., 2002). The OSVZ is located superficially to the ISVZ and shows a columnar organization of cells, whereas the ISVZ consists of randomly oriented cells. Interestingly, the thickness of the OSVZ increases during development, while that of the ISVZ is rather constant, indicating that the OSVZ is the major source of the cortical neurons, at least during late cortical plate (CP) development. Recently, Hansen et al. and Fietz et al. demonstrated the existence of a novel type of progenitor cell in the human OSVZ (Fietz et al., 2010; Hansen et al., 2010). This type of progenitor cells has a long ascending process and expresses stem cell markers such as Pax6, Hes1, and Sox2. During the mitosis of this cell type, the ascending process is maintained and is inherited by one of the daughter cells, which is then able to further divide in the same manner. Thus, this type of cells undergoes self-renewing asymmetric cell divisions. These morphological and behavioral features strikingly resemble those of RG in the VZ. Accordingly, Hansen et al. referred to these cells as OSVZ radial glia-like (oRG) cells. They originally postulated that oRG cells might have newly appeared during primate evolution. However, oRG cells were later also found in other mammalian species, including ferrets, rats and mice (Fietz et al., 2010; Reillo et al., 2011; Shitamukai et al., 2011; Wang et al., 2011; Kelava et al., 2012; Martínez-Cerdeño et al., 2012), and they were found not only in the OSVZ, but also in the ISVZ in ferrets and marmosets (Reillo et al., 2011; García-Moreno et al., 2012).
Therefore, this cell type is also called basal RG (bRG) (Englund et al., 2005). It is generally thought that the dramatic increase in the number of bRGs in the primate fetal cortex, especially in humans, may underlie the acquisition of a large cerebral cortex during evolution.

After the description of human OSVZ cells by Hansen et al., we noticed that the histological features of the mouse IZ/SVZ were well-correlated with the primate SVZ as follows (Tabata et al., 2012). First, the mouse MAZ and the primate ISVZ are both located just above the $\mathrm{VZ}$ and are composed of highly packed and randomly oriented cells. Second, both the mouse REP cells and the primate bRG cells have a long ascending process, exhibit proliferative activity, and are located widely in the SVZ/IZ from just above the VZ to the IZ. The mouse REP is not equal to mouse bRGs. Rather, REP includes BPs as the major population and lessor Olig2-positive glial progenitors in addition to the self-renewed mouse bRGs. All these cell types in the REP have a long ascending process, at least before the first cell division in the SVZ. The cells with this morphology, including Olig2-positive glial progenitors, are called translocating RG (tRG) (MartínezCerdeño et al., 2012). We observed that BPs among the REP also have the tRG morphology before cell division in the SVZ/IZ and that they were indistinguishable from bRG morphologically, suggesting that BPs and bRG might not be completely distinct cell types, but instead might only differ in the degree of their undifferentiated states. Hence, we hypothesized that the expansion of the SVZ during brain evolution in primates might have been caused by an increase in REP production and/or maintenance rates in primates, compared with that in rodents. Therefore, we assumed that the molecular mechanisms responsible for the regulation of the REP/SEP production ratio might have evolved and been promoted in primates in a manner such that the number of bRGs was increased.

In mice, we previously observed that the REP production rate, compared with the SEP production rate, was relatively high in the lateral cortex and low in the dorsomedial cortex (Tabata et al., 2009). This fact is probably due to the need of the lateral VZ to produce more neurons destined to reach the laterally extended CP outside the lateral edge of the cortical VZ. Whatever the reason for this difference in production rates in mice, we expected that genes regulating the higher REP/SEP production ratio in the mouse lateral VZ might be among the candidates genes responsible for the increased generation of bRGs in primate cortex, leading to the acquisition of a huge cerebral cortex, during evolution.

Here, we compared the expression profiles between lateral and dorsomedial VZ cells in mice during late cortical plate development. The gene expression profiles were analyzed using Affymetrix GeneChip microarrays. This screening of differentially expressed genes combined with validation using in-situhybridization experiments identified 29 and 9 genes with higher expression levels in the lateral and dorsomedial cortices, respectively. We then performed detailed bioinformatics evolutionary analyses. As a result, significant changes were found for six genes: the negative selection pressures on Megf11, Dmrt3, and Cntn3 were weaker in primate lineages than in non-primate lineages, whereas those on Jag1, Ntrk2, and Pmp22 were stronger in primate lineages than in non-primate lineages. Candidate molecules 
responsible for primate cortical expansion through increase of bRGs may be included in these genes.

\section{MATERIALS AND METHODS ANIMALS}

Pregnant ICR mice were purchased from Japan SLC. The day of vaginal plug detection was considered embryonic day 0 (E0). All the animal experiments were performed according to the Guidelines for the Care and Use of Laboratory Animals of Keio University School of Medicine.

\section{FLUORESCENCE-ACTIVATING CELL SORTING (FACS) OF THE LATERAL AND DORSOMEDIAL VZ CELLS}

To label the differentiation states of the lateral and dorsomedial VZ cells, we introduced a green fluorescent protein (GFP) expression vector at E15.5 using in utero electroporation. The vector we used expresses enhanced GFP (EGFP) cDNA under the control of the CAG promoter, which contains the cytomegalovirus early enhancer and chicken beta-actin promoter (a kind gift from Dr. Jun-ichi Miyazaki, Osaka University) (Niwa et al., 1991). In utero electroporation was performed as described previously (Tabata and Nakajima, 2001). Briefly, we injected $3 \mu \mathrm{g} / \mu \mathrm{L}$ of plasmid solution into the lateral ventricles and applied $35-\mathrm{V}, 50-\mathrm{ms}$ pulses 4 times using an electroporator (CUY-21E or NEPA21; NEPA GENE) with a forceps-type electrode (CUY650P5). To label the lateral and dorosomedial VZ cells differentially, we changed the direction of the electrode.

At $18 \mathrm{~h}$ after the electroporation, the brains were removed and the GFP-positive area was dissected out under a dissection microscope with a fluorescent lamp. The tissue fragments were treated with trypsin and DNaseI and then triturated by pipetting in DMEM with 10\% FCS. GFP-positive cells were isolated using EPICS ALTRA HyPerSort and Expo 32 software (Beckman Coulter). Dissociated cells were stained with propidium iodide (PI) prior to sorting. The gating parameters were determined using the side and forward scatter to eliminate debris and aggregated cells, using red fluorescence $(610 \mathrm{~nm})$ to eliminate dead cells, and using green fluorescence $(488 \mathrm{~nm})$ to separate the positive from negative cells (cells prepared from un-injected brains were used as the negative control).

The sorted cells were directly collected in Trizol (Invitrogen). We performed FACS 3 times independently and pooled all the samples, collecting about $5 \times 10^{5}$ cells for each sample in total. The total RNA was extracted from each sample. The quality control and an estimation of the extracted total RNA concentration were performed using an Agilent 2100 Bioanalyzer (Agilent Technologies).

\section{GENECHIP EXPRESSION ANALYSIS}

About $130 \mathrm{ng}$ of total RNA was used to prepare biotinylated cRNAs, which were hybridized to Affymetrix GeneChip Mouse Expression Set 4302.0 arrays. The GeneChip data analysis was performed as described previously (Tachikawa et al., 2008). Briefly, the hybridized microarray images were analyzed using the Affymetrix Microarray Software 5.0 (MAS5.0) algorithm. The detection calls, change calls, and the signal log ratios were determined using the Expression Console Version 1.1 (Affymetrix).
We classified the candidate genes using gene ontologies (GOs) and the Protein Analysis Through Evolutionary Relationships (PANTHER) database analysis software (SRI International, Menlo Park, CA, http://www.pantherdb.org).

\section{In situ HYBRIDIZATION}

E16 ICR mouse embryos were fixed using perfusion with 4\% paraformaldehyde (PFA) in $0.1 \mathrm{M}$ sodium phosphate buffer (pH7.4). The brains were removed and post-fixed in the same fixative overnight at $4^{\circ} \mathrm{C}$. The brains were then equilibrated in 30\% sucrose in PBS and embedded in OCT compound (Sakura Finetek). Frozen sections were cut coronally in 20$\mu \mathrm{m}$ thick sections using a cryostat (CM1900; Leica) and were mounted onto MAS-coated glass slides (Matsunami). To prepare the RNA probes, the plasmid vectors of the RIKEN FANTOM clone set were digested with appropriate restriction enzymes or PCR products amplified from the RIKEN FANTOM clone set using a primer pair (forward primer M1321, 5'-TGTAAAACGACGGCCAGT-3'; reverse primer $1233,5^{\prime}$ AGCGGATAACAATTTCACACAGGA- $3^{\prime}$ ) as the template for in vitro transcription. The clones that were used are listed in Table 1. For Dmrt3, we used GeneCopoeia GC-Mm23549 as the template DNA. Digoxigenin (DIG)-labeled RNA probes were synthesized using T7 or T3 RNA polymerase and the DIG RNA labeling kit (Roche). Sections were treated with 4\% PFA and acetylated with 100-mM triethanolamine ( $\mathrm{pH} 8.0$ ). Pre-hybridization was performed in $50 \%$ formamide, $5 \mathrm{x}$ SSPE, $5 \%$ SDS, and $1 \mathrm{mg} / \mathrm{mL}$ of yeast transfer RNA (Sigma) at $55^{\circ} \mathrm{C}$ for $1 \mathrm{~h}$. Hybridization was performed in the same solution with $1 \mu \mathrm{g} / \mathrm{mL}$ of a DIG-labeled RNA probe at $55^{\circ} \mathrm{C}$ overnight. The hybridized probes were reacted with alkaline phosphatase-conjugated anti-DIG antibody (1:2000; Roche). Signals were developed in alkaline phosphatase buffer containing nitroblue tetrazolium chloride (NBT) and 5bromo-4-chloro-3-indolyl-phosphate (BCIP).

\section{BIOINFORMATICS EVOLUTIONARY ANALYSES}

We performed bioinformatics evolutionary analyses for 38 candidate genes that are differentially expressed between lateral and dorsomedial VZ cells in mice. To determine the genes of other species that are orthologous to these 38 candidate genes in mice, one-to-one orthologous relationships were obtained from the EnsemblCompara DB (Vilella et al., 2009). We accepted only one-to-one orthologous relationships so as to avoid the inclusion of paralogous genes in our evolutionary analyses. We compared the coding sequences (CDS) of genes in 13 mammals (human, chimpanzee, orangutan, macaque, mouse, rat, rabbit, dog, cow, armadillo, elephant, tenrec, and opossum) as well as those of the anole lizard as an out-group. Although genome information for other vertebrates is available, we did not include such species because of poor-quality genome assembly and/or gene annotations. Reliable genomic information was selected according to the "13 eutherian mammals EPO" dataset in Ensembl (http://asia. ensembl.org/info/docs/compara/analyses.html).

Given the CDS of orthologous genes in the 14 species, multiple alignments were calculated using PRANK+F (Löytynoja and Goldman, 2008) based on the phylogenetic tree shown in Figure 3A. Alignment columns containing gaps were removed, as 


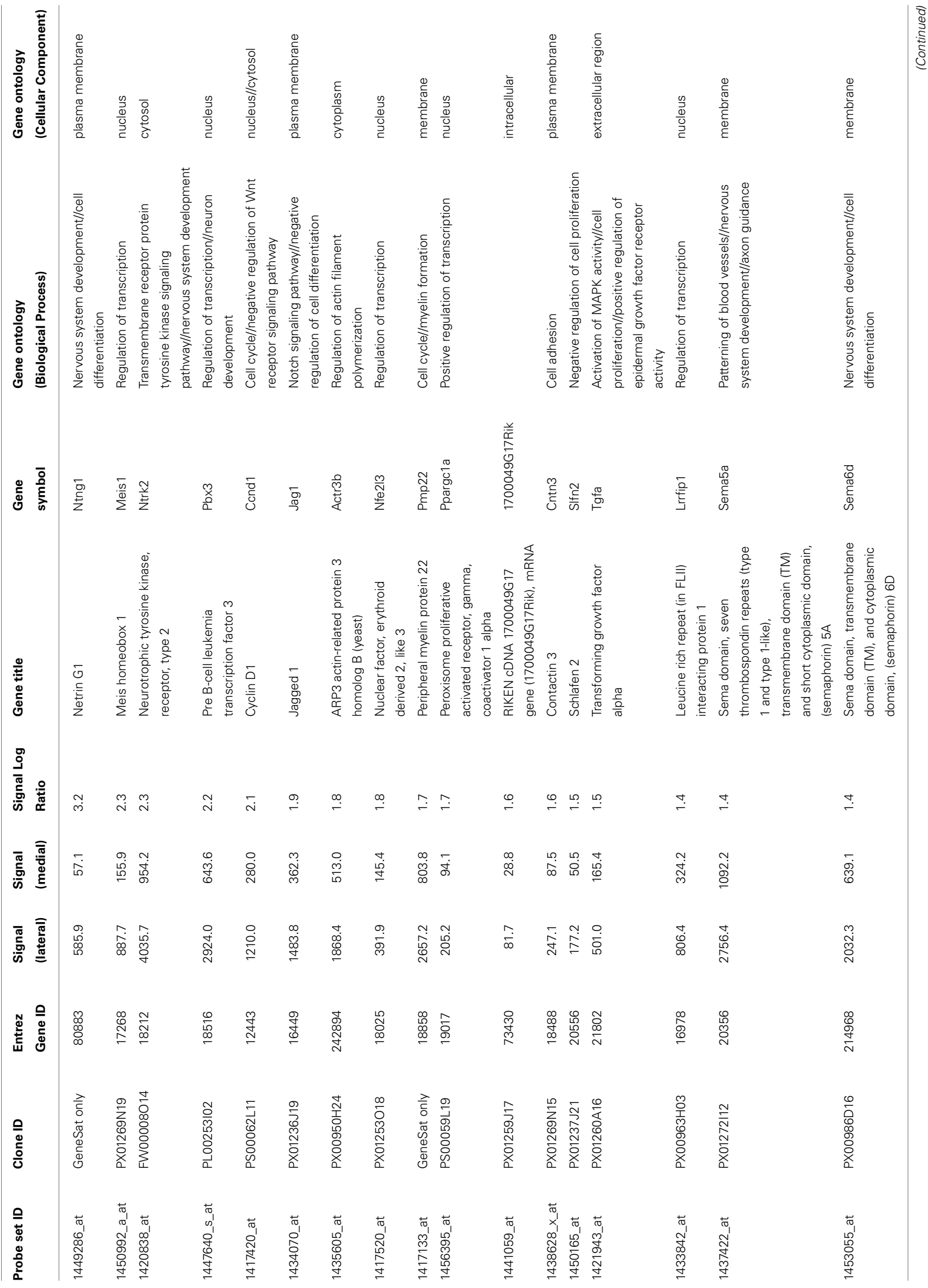




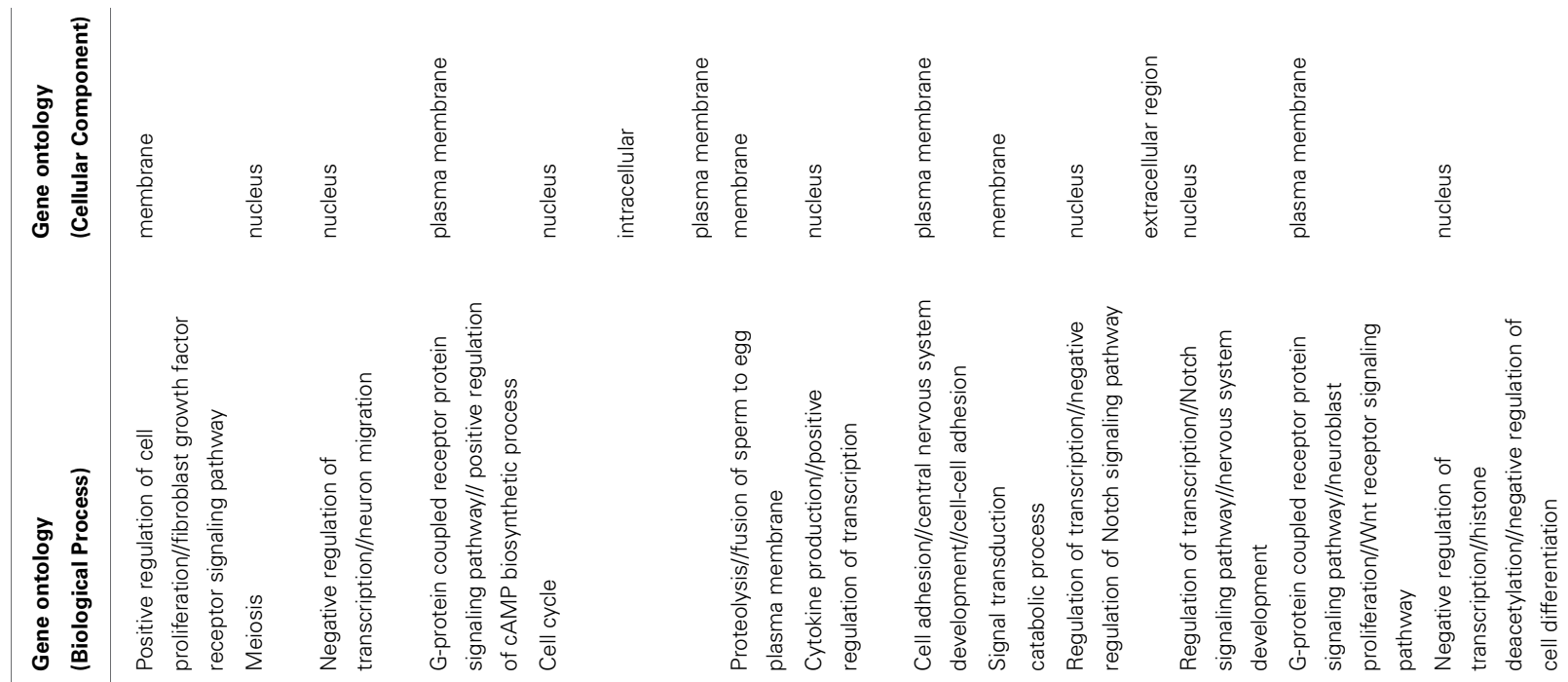

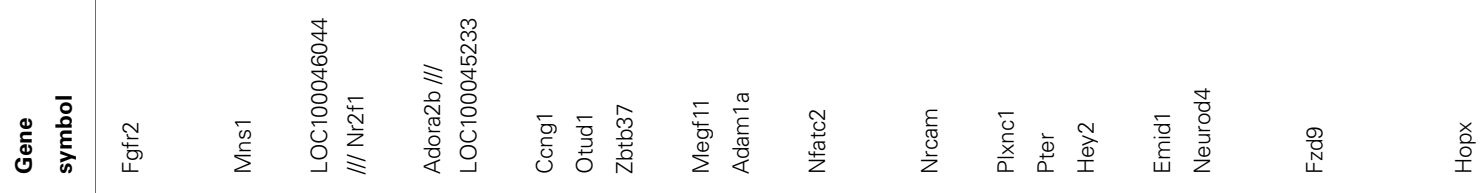

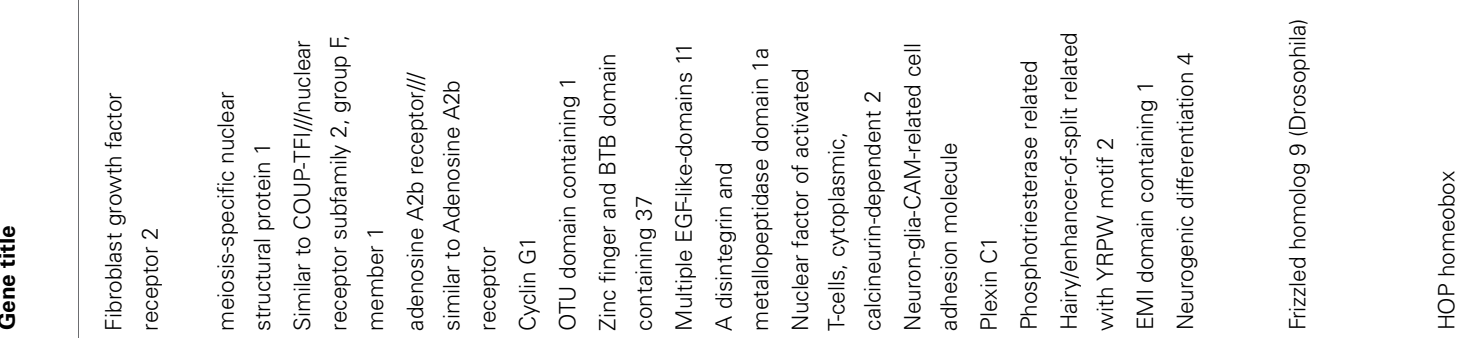

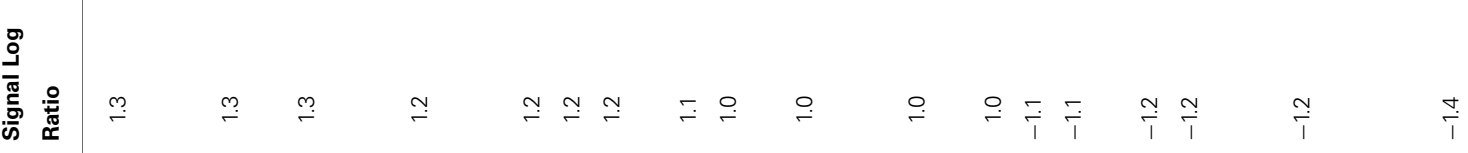

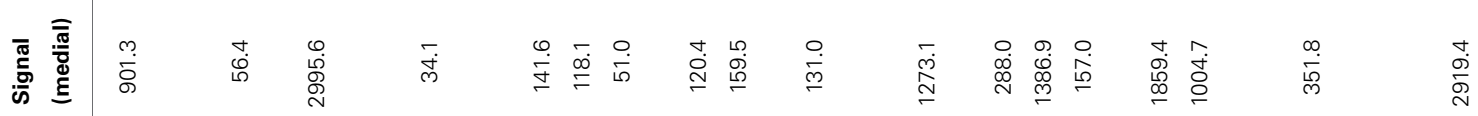

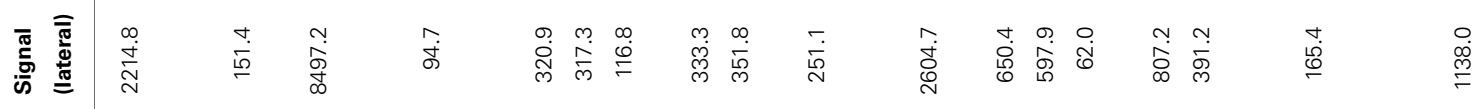

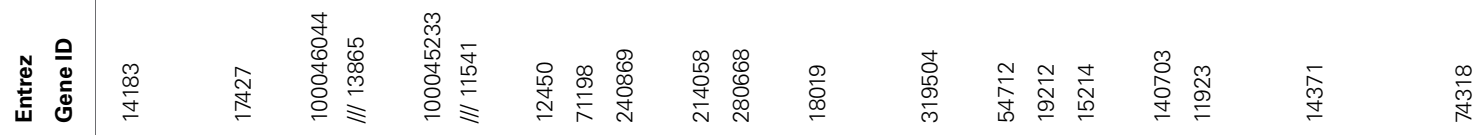

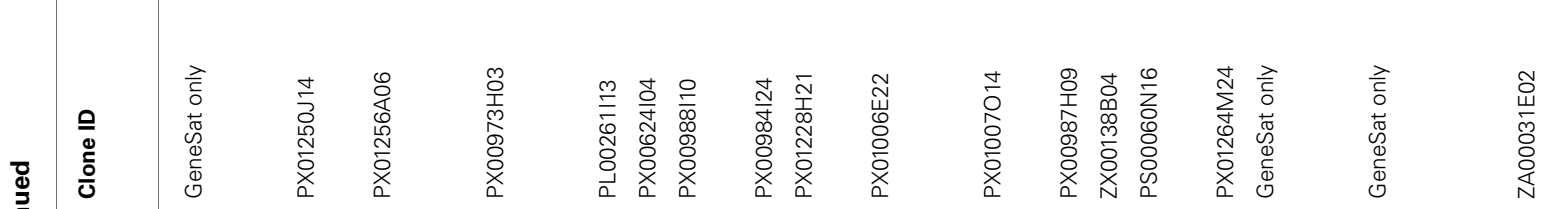

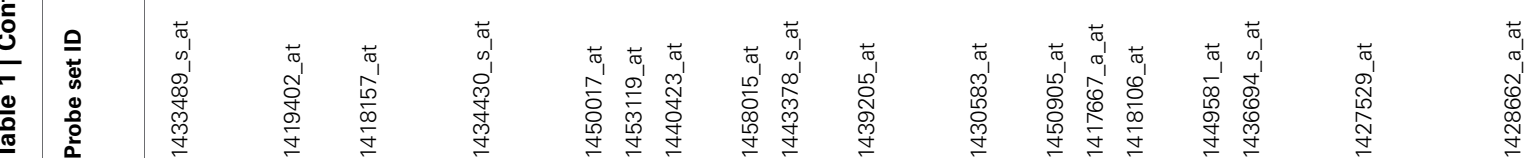




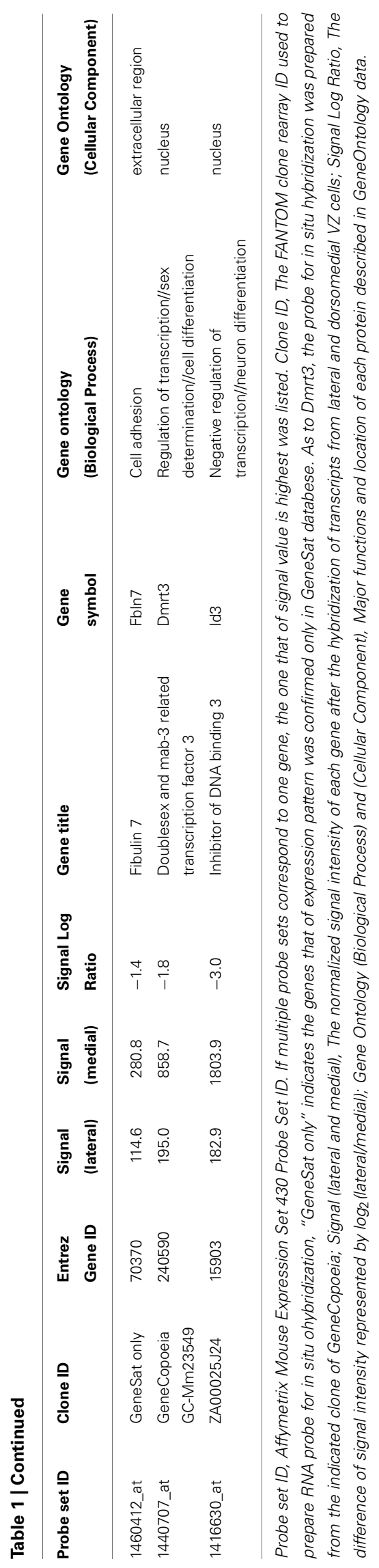

described previously (Tárraga et al., 2007; Capella-Gutiérrez et al., 2009).

To elucidate the evolutionary pressure on the candidate genes, we estimated the $\mathrm{dN} / \mathrm{dS}$ ratios based on evolutionary models. The first model contained two $\omega(=\mathrm{dN} / \mathrm{dS})$ parameters (Yang, 1998): one $\omega$ was for primate lineages, and the other was for nonprimate lineages. This means that the $\mathrm{dN} / \mathrm{dS}$ ratio was constant among primate and non-primate lineages, respectively. Because the model uses two $\omega(=\mathrm{dN} / \mathrm{dS})$ parameters, the $\mathrm{dN} / \mathrm{dS}$ ratios can differ between primate and non-primate lineages. The model also assumed that the $\omega$ ratio was constant across whole amino acid residues of a gene product. We denoted this evolutionary model as the "two-ratio" model.

To test whether the $\mathrm{dN} / \mathrm{dS}$ ratio of primate lineages differs significantly from that of non-primate lineages, an alternative evolutionary model with one $\omega$ parameter was also used (Yang, 1998). This model assumed that the $\mathrm{dN} / \mathrm{dS}$ ratio was constant for all lineages of the phylogenetic tree shown in Figure 3A and that the $\mathrm{dN} / \mathrm{dS}$ ratio was constant across whole amino acid residues of a gene product. This model was called the "one-ratio" model. To determine which of the one-ratio or the two-ratio models had a better fit with a given alignment, a likelihood ratio test (Yang, 1998) was applied. If the two-ratio model was accepted, this finding would suggest that the $\mathrm{dN} / \mathrm{dS}$ ratio of primate lineages differed significantly from that of non-primate lineages. The calculation of the likelihood ratio test was conducted using PAML4 (Yang, 2007).

For detailed evolutionary analyses, we employed branch-site models, which relax the assumption of the above one-ratio and two-ratio models and allow the $\omega$ ratio to vary both among lineages and among amino acid sites (Yang and Nielsen, 2002; Yang et al., 2005; Zhang et al., 2005). The branch-site test of positive selection was applied to given alignments as described in PAML (Yang, 2007). Amino acid residues under positive selection were predicted using the Bayes empirical Bayes (BEB) method (Yang et al., 2005). The calculation of the branch-site test of positive selection was executed by PAML4 (Yang, 2007).

\section{RESULTS \\ SCREENING OF LATERALLY AND MEDIALLY EXPRESSED GENES IN MICE DURING LATE CORTICAL PLATE DEVELOPMENT}

Because the ratio of the production of proliferative cells in the mouse IZ/SVZ (REP) to that of postmitotic cells (SEP) within the population exiting from the VZ is high in the lateral $\mathrm{VZ}$ and low in the dorsomedial VZ (Tabata et al., 2009), we compared the gene expression profiles between the lateral $\mathrm{VZ}$ and the dorsomedial VZ to screen for candidate genes regulating the REP/SEP production ratio in mouse cortex. We first introduced a GFP expression vector into the lateral or dorsomedial $\mathrm{VZ}$ at embryonic day (E) 15, and GFP-positive cells were collected by FACS $18 \mathrm{~h}$ later, by which time the fate of newly born cells in the VZ (whether they will belong to the REP or SEP) is thought to have been determined (Tabata et al., 2009) (Figure 1). We extracted RNA from the GFP-positive cells and performed transcriptome analyses using DNA microarrays. We selected probe sets with the detection call "present" $(P<0.05)$ in both lateral and dorsomedial VZ cells, since we had previously noticed that the REP production rate was 


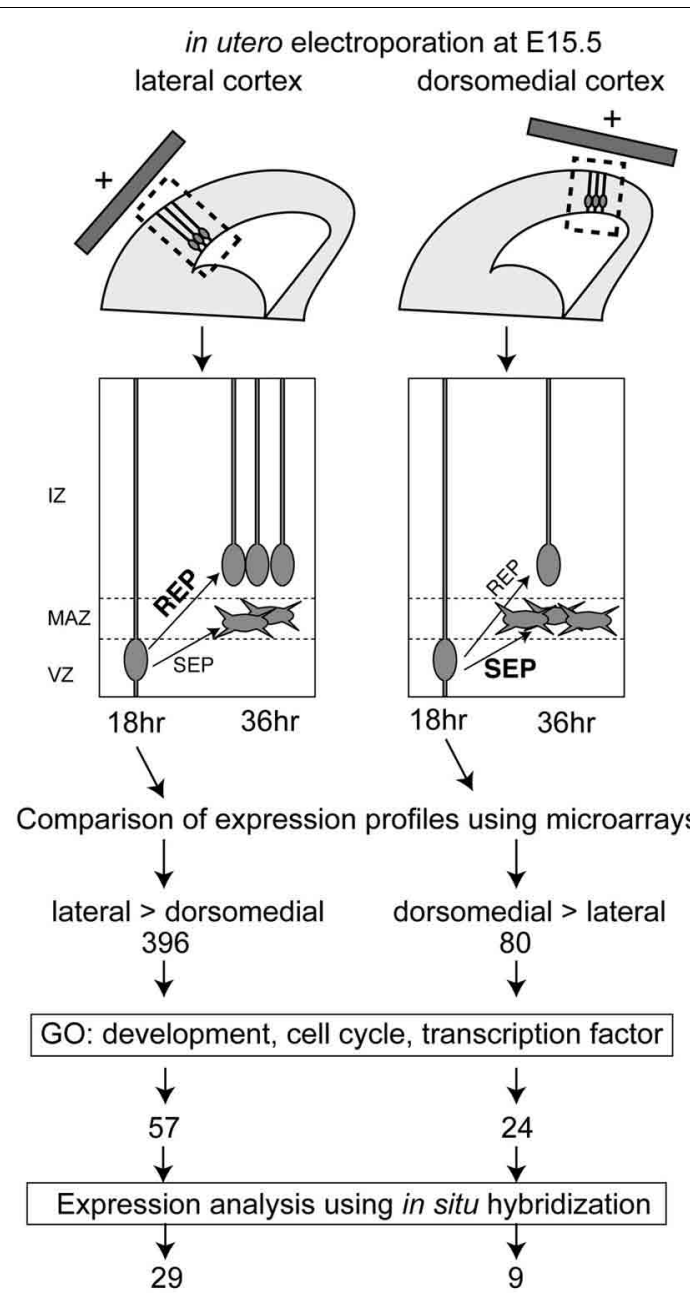

FIGURE 1 | Screening procedures for identifying genes involved in the regulation of the REP production rate and the number of genes selected in each step. The REP production rate was high in the lateral VZ, compared with the dorsomedial region. Hence, the lateral and dorsomedial VZ cells labeled using GFP and in utero electroporation at E15.5 were collected $18 \mathrm{~h}$ after electroporation, when the cells undergo differentiation into REP or SEP. Microarray analyses identified 396 genes with a lateral-high and 80 genes with a medial-high expression pattern. The candidate genes were further selected based on gene ontology (GO) and were narrowed down to 62 candidate genes with lateral-high and 23 candidate genes with medial-high expression patterns. The expression patterns of these candidate genes were checked in the BGEM database or by performing in situ hybridization, and 29 genes with lateral-high and 9 genes with medial-high expression patterns were identified.

low in the dorsomedial region but remained significant [the REP production rate among cells exiting the $\mathrm{VZ}$ was about $65 \%$ in the lateral VZ while that in the dorsomedial VZ was $40 \%$ when labeled at E15 (Tabata et al., 2009)]. We further selected probes for which the signals showed a high lateral, low dorsomedial pattern or the opposite pattern with an increased or decreased call $(P<0.05)$ using the MAS5 algorithm, followed by those with a threshold of a 2 -fold change ( 1 log-fold change). As a result, we identified 396 and 80 probe sets with high lateral and high dorsomedial expression patterns, respectively. We then obtained the gene ontologies (GOs) for the probe sets and narrowed down the candidates to those with a GO related to development or the cell cycle. The reason why we chose a "cell cycle" GO is that since the SEP is postmitotic, whereas the REP is still proliferative, such a GO would be expected for genes involved in the decision to acquire an SEP fate or an REP fate among the VZ-derived cells. We also selected transcription factors to obtain potential key factors for the REP/SEP production ratio. In this step, we integrated multiple probe sets into one transcript. Using these procedures, we identified 62 and 23 genes with higher expressions in the lateral and dorsomedial $\mathrm{VZ}$ regions, respectively.

Next, we examined the expression profiles of these 85 candidate genes using an in-situ hybridization database, the Brain Gene Expression Map (BGEM; http://www.stjudebgem. org/web/mainPage/mainPage.php) (Magdaleno et al., 2006). Consequently, we found 5 lateral-high ( $F g f r 2, N t r k 2, P b x 3$, Pmp22, Ntng1) and 5 dorsomedial-high (Dmrt3, Id3, NeuroD4, $F z d 9, F b \ln 7)$ candidate genes in the BGEM database. In-situ hybridization experiments included in the BGEM database showed gradient expression patterns for E15.5 mouse forebrain that were consistent with our microarray results, except for those for the $F b \ln 7$ gene (data not shown). For 2 lateral-high genes (Ntrk2 and Pbx3) and 2 medial-high genes (Dmrt3 and Id3), for which the expression patterns shown in the BGEM database appeared to be consistent with our microarray results, we further confirmed the expression gradient in the VZ using in situ hybridization with the RIKEN FANTOM cDNA clone set (Okazaki et al., 2002; Carninci et al., 2005) as templates for probe synthesis (Figures 2A,B).

For other candidates, we performed in situ hybridization to confirm the gradient expression in the VZ. Among the remaining 57 lateral-high and 18 medial-high genes, 49 and 16 genes were included in the cDNA clone set with an insert longer than 1 kilobase pairs (kbp). We therefore performed in situ hybridization for these genes on sections of E16 mouse brains and found 24 lateralhigh and 4 medial-high candidate genes. One lateral-high gene, Sema6d, showed a lateral-high to medial-low gradient only in the caudal region (Figure 2Am).

Overall, we identified 29 and 9 genes expressed in a lateral-high to medial-low or a medial-high to lateral-low gradient pattern in the VZ, respectively (Figure 1).

In this screening, we did not obtain well-known arealization factors (Borello and Pierani, 2010) as candidate genes. Among such genes, we were able to detect the expressions of Pax6, Emx2, Bmp2, and Wnt5a in both the lateral and dorsomedial VZ cells, but the differences in the expression levels between the lateral and dorsomedial VZs were not significant according to the MAS5.0 algorithm. As for Fgf8, Bmp4, and Wnt3a, the expressions of these genes were not detected in either the lateral or dorsomedial VZ cells. This observation might reflect the fact that we collected the VZ cells at a late stage of cortical plate development, at which time arealization had already been completed.

\section{BIOINFORMATICS EVOLUTIONARY ANALYSIS ON THE LATERAL-HIGH AND MEDIAL-HIGH CANDIDATE GENES}

The 29 and 9 genes with lateral-high and medial-high expression patterns, respectively, can be considered as potential candidates 


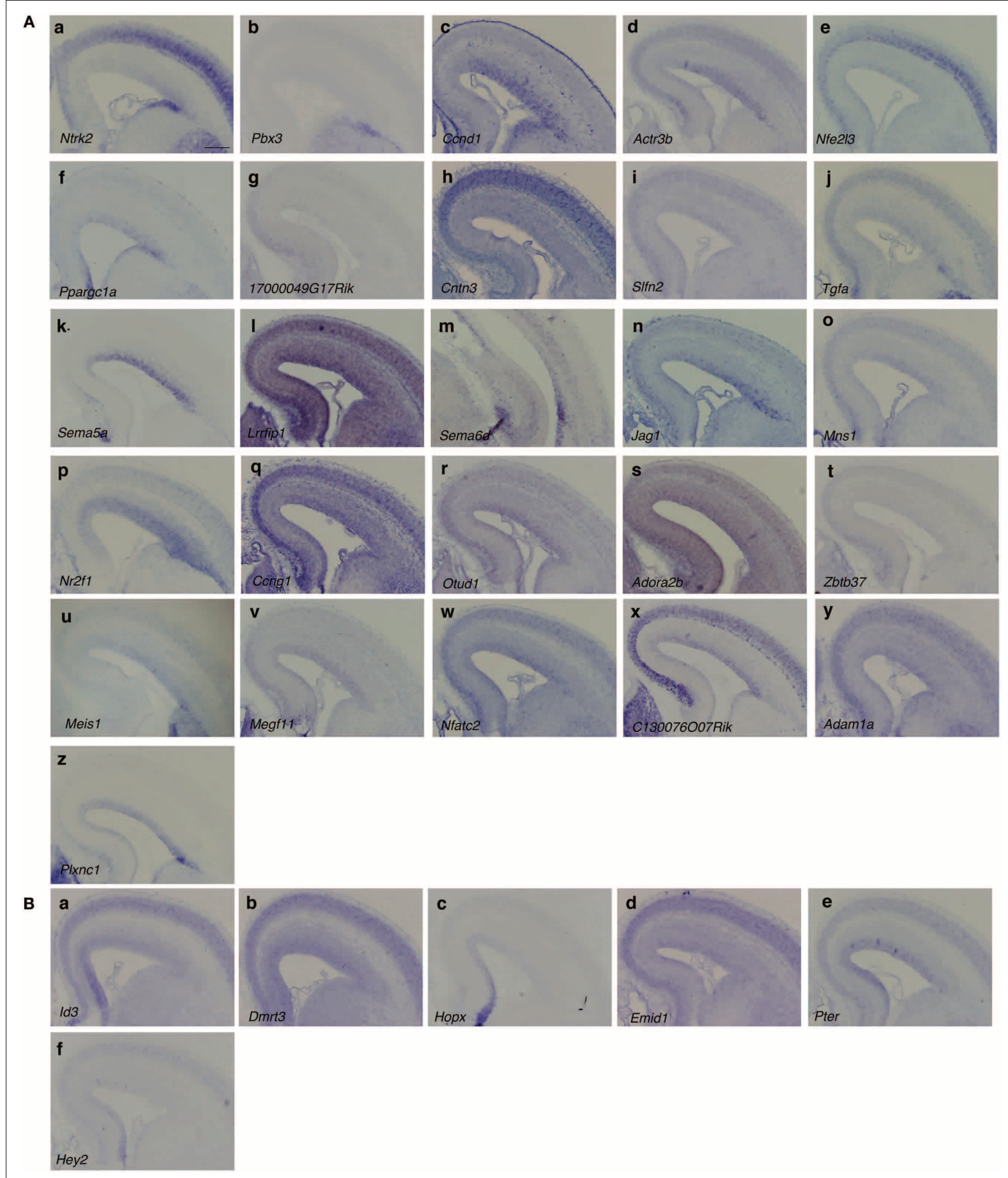

FIGURE 2 | In situ hybridization of candidate genes. The DIG-labeled RNA probes of the indicated genes were hybridized on the coronal section of E16 mouse brains. Genes with (A) lateral-high and (B) medial-high expression patterns are shown. Scale bar: $200 \mu \mathrm{m}$. 
responsible for the high REP/SEP production ratio in the mouse lateral cortex, compared with the dorsomedial cortex. These genes might include candidate molecules that were involved in the dramatic increase in the number of bRGs during primate cortical evolution. Thus, we then performed bioinformatics evolutionary analyses for these 38 genes. The evolutionary pressures on the candidate genes were estimated using evolutionary models, which are based on the synonymous and non-synonymous base substitution rates $(\mathrm{dN} / \mathrm{dS})$ of the coding DNA sequences.

To estimate the $\mathrm{dN} / \mathrm{dS}$ ratios, we first investigated the one-toone correspondence of candidate genes among 13 mammalian species and 1 reptile, the Anole lizard, as shown in Figure 3A.
A

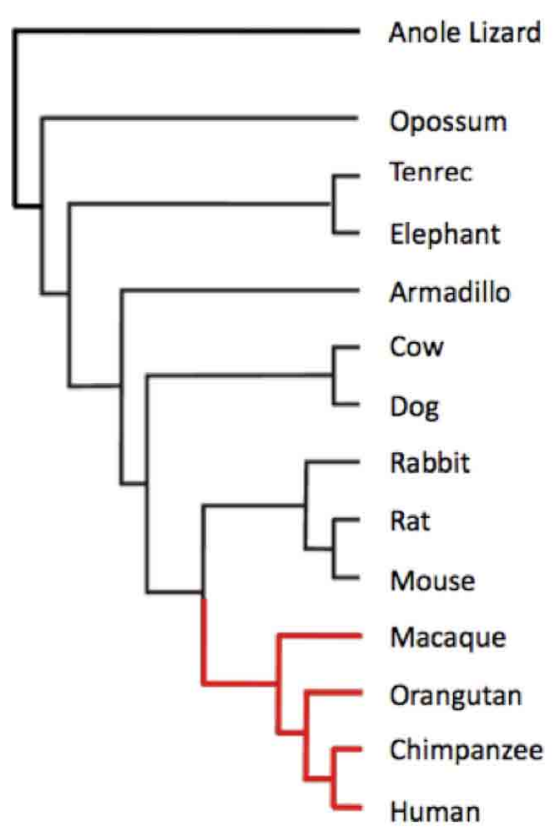

B

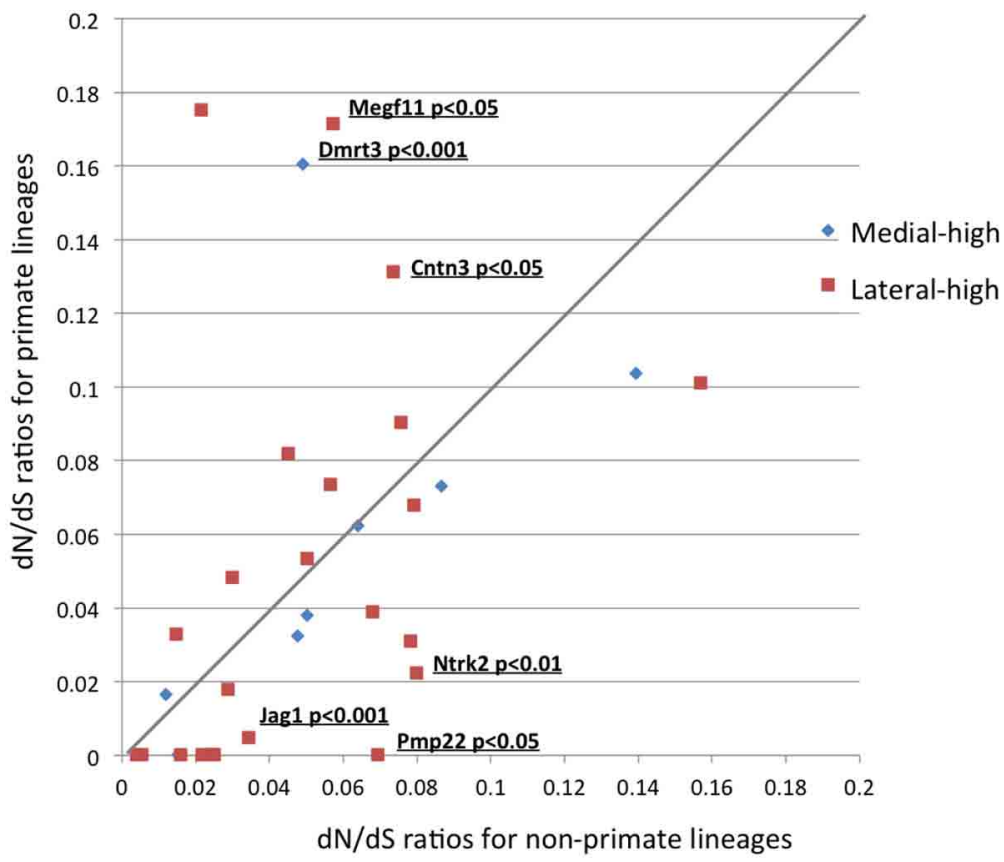

C

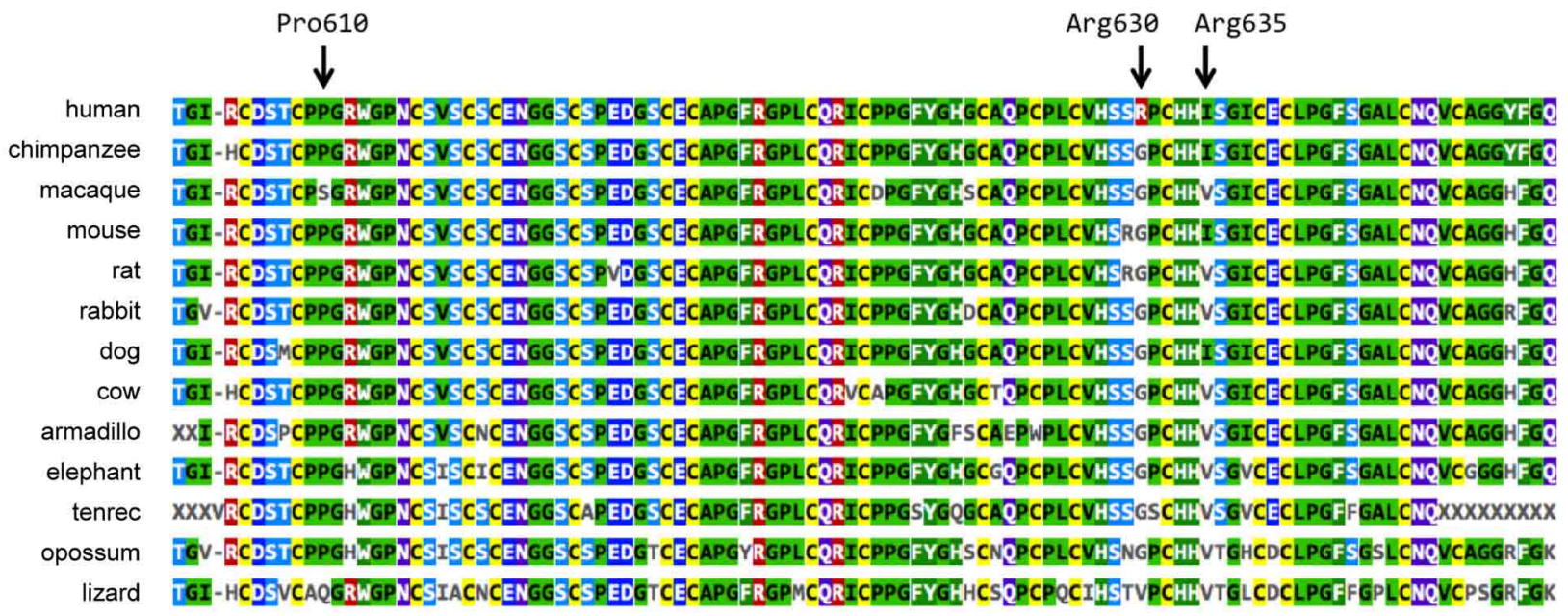

FIGURE 3 | Bioinformatics evolutionary analyses of 38 candidate genes. (A) Phylogenetic tree used to perform the multiple alignments. The red and black lineages in the phylogenetic tree represent primate and non-primate lineages, respectively. (B) Two-dimensional plotting of the dN/dS ratios in primate and non-primate lineages. The $\mathrm{X}$ - and $\mathrm{Y}$-axes represent $\mathrm{dN} / \mathrm{dS}$ ratios for non-primate and primate lineages, respectively. The gene symbol and $P$-values are shown for differences between primate and non-primate lineages with a significance level of 0.05 . (C) Alignment of the Megf11 protein sequences in 13 species. The orangutan Megf11 protein sequence was not included in this alignment because Ensembl did not contain any Megf11 gene annotation for the orangutan genome, possibly because of the low quality of the genome. This alignment shows that Arg630 is a human-specific residue while the amino acid site is strongly conserved among mammalian species, except for humans. 
We excluded three candidate genes (Slfn2, 1700049G17Rik, and Adam1a) from subsequent evolutionary analyses because these genes were found to have undergone gene loss or gain events when mice and humans were compared.

To figure out evolutionary pressures of the remaining 35 candidate genes, we applied the "two-ratio" evolutionary model (see Materials and Methods) to the candidate genes. Figure 3B shows the $\mathrm{dN} / \mathrm{dS}$ ratios of the 35 candidate genes in primate (y-axis) and non-primate ( $\mathrm{x}$-axis) lineages. The likelihood ratio test, which compares the "one-ratio" and "two-ratio" evolutionary models, suggested that the dN/dS ratios of three genes (Jag1, Ntrk2, and Pmp22) were significantly lower in primate lineages than in non-primate lineages $(P<0.05)$. These results indicated that the negative selection for Jag1, Ntrk2, and Pmp22 in the primate lineages was stronger than that in the non-primate lineages, implying that the molecular functions of these three genes are likely to be more essential in primates than in non-primates. In contrast, the dN/dS ratios of three other genes (Megf11, Dmrt3, and $C n t n 3)$ were significantly higher in the primate lineages, compared with those in the non-primate lineages $(P<0.05)$.

Next, we performed additional detailed evolutionary analyses for Megf11, Dmrt3, and Cntn3. dN/dS ratios higher than 1.0 are indicative of adaptive molecular evolution (Hughes and Nei, 1988; Messier and Stewart, 1997; Zhang, 2003). Although the $\mathrm{dN} / \mathrm{dS}$ ratios averaged over the whole amino acid residues of the Megfl1, Dmrt3 and Cntn3 gene products were less than 1.0 , we searched for amino acid residues with positive selection in primate lineages using branch-site evolutionary models (see Materials and Methods). For Dmrt3 and Cntn3, our branchsite models yielded no residues under positive selection, i.e., the $\mathrm{dN} / \mathrm{dS}$ ratios of all the residues were estimated to be smaller than 1.0. For Megf11, the branch-site models suggested that seven residues (Arg561, Pro568, Pro610, Arg630, Ile635, Ser802 and Met971) were under positive selection in primate lineages. Among the seven residues, Arg630 is human-specific (Figure 3C), Arg561 is chimpanzee-specific, Pro568 and Pro610 are macaquespecific, Ile635 is common among humans and chimpanzees, and Ser802 and Met971 are common among primates. Arg630 is harbored in the laminin-type EGF-like domain, which mediates cell adhesion, growth, migration and differentiation. These results suggested that Megf11 underwent adaptive molecular evolution in primate lineages, implying that the molecular function of Megfl1 might be involved in primate-specific traits. Among the three genes with $\mathrm{dN} / \mathrm{dS}$ ratios that differed significantly between primate and non-primate lineages, Jag1 showed the most obvious primate-specific selection $(P<0.001)$.

\section{DISCUSSION}

In this study, we attempted to identify candidate molecules that were potentially involved in the expansion of the cerebral cortex during primate evolution. We previously reported that the ratio of REP production to SEP production in the mouse cortex was higher in the lateral VZ than in the dorsomedial VZ and that the mouse REP had characteristics similar to those of primate bRG cells. Specifically, both have a long ascending process and are distributed widely in the SVZ, overlapping with or superficially to the region with a high cell density located above the VZ (MAZ in mouse and ISVZ in primates), which is composed of randomly oriented cells. Moreover, mouse REP includes Sox2-positive and/or Pax6-positive bRG-equivalent cells as a minor population, in addition to BPs and glial progenitors. Based on these facts, we assumed that the molecules responsible for the high REP/SEP production ratio in the mouse lateral cortex, compared with the dorsomedial cortex, might include candidate genes that had contributed to the expansion of bRGs in the primate cortex during evolution. Therefore, we screened for genes that were expressed in a lateral-medial gradient pattern to identify candidate molecules involved in the determination of the REP/SEP production ratio in mouse cortex. We then further performed detailed evolutionary analyses of the resulting candidate genes. As a result, significant changes in evolutionary pressure between primate and non-primate lineages were found for six genes. These genes are known/suggested to be involved in cell proliferation, differentiation, and adhesion and might affect the REP/SEP production ratio in mice.

Of course, some of the candidate genes that were isolated might have simply reflected areal differences. Further analyses are needed to test the roles of candidate genes on the regulation of the REP/SEP production ratio. During cortical development, a neurogenic gradient exists from the lateral to dorsomedial region. Hence, the difference in the REP/SEP production ratio between the lateral cortex and the dorsomedial cortex might reflect differences in the developmental stage. However, we previously showed that the REP production rates of the VZ cells labeled at E15 and E16 were not dramatically different from each other (65 and 68\%, respectively) (Tabata et al., 2009). Therefore, we assumed that the higher REP/SEP production ratio in the lateral cortex, compared with that in the dorsomedial cortex, was unlikely to be simply due to the differences in the neurogenic stages, but to have been largely caused by the need to satisfy the regional demand for neuron production.

The significance of Notch signaling in the proliferation of bRG cells has been previously reported (Hansen et al., 2010). Considering that the mouse REP is a proliferative population, while the SEP is a postmitotic population, Jag1, a conventional ligand of Notch receptors, is an important candidate that might have evolved to increase the REP/SEP production ratio in primates. Megf11 is a member of the MEGF family, which includes Megf10 and Jedi-1 in mammals and CED-1 in C. elegans. Although MEGF family proteins were initially shown to mediate engulfment as a binding protein for cell corpses (Zhou et al., 2001; Wu et al., 2009), a later study demonstrated that MEGF family proteins also act as a Notch signaling modifier (Krivtsov et al., 2007). MEGF family proteins have a weak homology to conventional Notch ligands, such as Jag1, including an N-terminal DSL-like domain and multiple EGF-like repeats. Krivtsov et al. demonstrated that MEGF family proteins inhibit Notch signaling, probably by binding directly to Notch receptors. The positive selection residues of the Megfl1 gene product were mainly harbored in the 11-14th EGF-like repeat. Thus, it would be interesting if the affinity with Notch receptors is changed in primates. Further analyses are needed to clarify the meaning of the primate-specific positive selections on the Megf11 gene. 
The role of the genes identified here, especially on the potential regulation of the REP/SEP production ratio, in mice and whether evolutionary changes in these genes were involved in the expansion of the OSVZ in primates should be clarified in future studies.

\section{ACKNOWLEDGMENTS}

We would like to thank J. Miyazaki (Osaka University, Osaka, Japan) for the CAG promoter, and the staff members at the Core
Instrumentation Facility, Keio University School of Medicine for FACS and GeneChip analyses, and H. Arai for technical assistance. This work was supported by the Strategic Research Program for Brain Sciences ("Understanding of molecular and environmental bases for brain health"), and Grants-in-Aid for Scientific Research of the Ministry of Education, Culture, Sports, and Science and Technology of Japan, Keio Gijuku Academic Development Funds, and Keio University Special Grant-in-Aid for Innovative Collaborative Research Projects.

\section{REFERENCES}

Borello, U., and Pierani, A. (2010). Patterning the cerebral cortex: traveling with morphogens. Curr. Opin. Genet. Dev. 20, 408-415. doi: 10.1016/j.gde.2010.05.003

Boulder Committee. (1970). Embryonic vertebrate central nervous system: revised terminology. Anat. Rec. 166, 257-261. doi: 10.1002/ar.1091660214

Capella-Gutiérrez, S., Silla-Martínez, J. M., and Gabaldón, T. (2009). trimAl: a tool for automated alignment trimming in largescale phylogenetic analyses. Bioinformatics 25, 1972-1973. doi: 10.1093/bioinformatics/btp348

Carninci, P., Kasukawa, T., Katayama, S., Gough, J., Frith, M. C., Maeda, N., et al. (2005). The transcriptional landscape of the mammalian genome. Science 309, 1559-1563. doi: $10.1126 /$ science.1112014

Englund, C., Fink, A., Lau, C., Pham, D., Daza, R. A. M., Bulfone, A., et al. (2005). Pax6, Tbr2, and Tbr1 are expressed sequentially by radial glia, intermediate progenitor cells, and postmitotic neurons in developing neocortex. J. Neurosci. 25, 247-251. doi: 10.1523/JNEUROSCI.2899-04. 2005

Fietz, S. A., Kelava, I., Vogt, J., WilschBräuninger, M., Stenzel, D., Fish, J. L., et al. (2010). OSVZ progenitors of human and ferret neocortex are epithelial-like and expand by integrin signaling. Nat. Neurosci. 13, 690-699. doi: 10.1038/nn. 2553

Gal, J. S., Morozov, Y. M., Ayoub, A. E., Chatterjee, M., Rakic, P., and Haydar, T. F. (2006). Molecular and morphological heterogeneity of neural precursors in the mouse neocortical proliferative zones. J. Neurosci. 26, 1045-1056. doi: 10.1523/JNEUROSCI.4499-05.2006 García-Moreno, F., Vasistha, N. A., Trevia, N., Bourne, J. A., and Molnár, Z. (2012). Compartmentalization of cerebral cortical germinal zones in a lissencephalic primate and gyrencephalic rodent. Cereb. Cortex
22, 482-492. doi: 10.1093/cercor/ bhr312

Hansen, D. V., Lui, J. H., Parker, P. R. L., and Kriegstein, A. R. (2010). Neurogenic radial glia in the outer subventricular zone of human neocortex. Nature 464, 554-561. doi: 10.1038/nature08845

Hughes, A. L., and Nei, M. (1988). Pattern of nucleotide substitution at major histocompatibility complex class I loci reveals overdominant selection. Nature 335, 167-170. doi: $10.1038 / 335167 \mathrm{a} 0$

Kelava, I., Reillo, I., Murayama, A. Y., Kalinka, A. T., Stenzel, D., Tomancak, P., et al. (2012). abundant occurrence of basal radial glia in the subventricular zone of embryonic neocortex of a lissencephalic primate, the common marmoset Callithrix jacchus. Cereb. Cortex 22, 469-481. doi: 10.1093/ cercor/bhr301

Konno, D., Shioi, G., Shitamukai, A., Mori, A., Kiyonari, H., Miyata, T., et al. (2007). Neuroepithelial progenitors undergo LGN-dependent planar divisions to maintain selfrenewability during mammalian neurogenesis. Nat. Cell Biol. 10, 93-101. doi: 10.1038/ncb1673

Krivtsov, A. V., Rozov, F. N., Zinovyeva, M. V., Hendrikx, P. J., Jiang, Y., Visser, J. W. M., et al. (2007). Jedi-a novel transmembrane protein expressed in early hematopoietic cells. J. Cell. Biochem. 101, 767-784. doi: 10.1002/jcb.21232

Löytynoja, A., and Goldman, N. (2008). Phylogeny-aware gap placement prevents errors in sequence alignment and evolutionary analysis. Science 320, 1632-1635. doi: 10.1126/science. 1158395

Magdaleno, S., Jensen, P., Brumwell, C. L., Seal, A., Lehman, K., Asbury, A., et al. (2006). BGEM: an in situ hybridization database of gene expression in the embryonic and adult mouse nervous system. PLoS Biol. 4:e86. doi: 10.1371/journal. pbio.0040086

Martínez-Cerdeño, V., Cunningham, C. L., Camacho, J., Antczak, J. L., Prakash, A. N., Cziep, M. E., et al. (2012). Comparative analysis of the subventricular zone in rat, ferret and macaque: evidence for an outer subventricular zone in rodents. PLoS ONE 7:e30178. doi: 10.1371/journal.pone.0030178

Messier, W., and Stewart, C. B. (1997). Episodic adaptive evolution of primate lysozymes. Nature 385, 151-154. doi: 10.1038/385151a0

Niwa, H., Yamamura, K., and Miyazaki, J. (1991). Efficient selection for high-expression transfectants with a novel eukaryotic vector. Gene 108, 193-199. doi: 10.1016/03781119(91)90434-D

Noctor, S. C., Flint, A. C., Weissman, T. A., Dammerman, R. S., and Kriegstein, A. R. (2001). Neurons derived from radial glial cells establish radial units in neocortex. Nature 409, 714-720. doi: 10.1038/ 35055553

Noctor, S. C., Martínez-Cerdeño, V., Ivic, L., and Kriegstein, A. R. (2004). Cortical neurons arise in symmetric and asymmetric division zones and migrate through specific phases. Nat. Neurosci. 7, 136-144.

Okazaki, Y., Furuno, M., Kasukawa, T., Adachi, J., Bono, H., Kondo, S., et al. (2002). Analysis of the mouse transcriptome based on functional annotation of 60,770 full-length cDNAs. Nature 420, 563-573. doi: 10.1038 /nature01266

Reillo, I., de Juan Romero, C., GarcíaCabezas, M. Á., and Borrell, V. (2011). A role for intermediate radial glia in the tangential expansion of the mammalian cerebral cortex. Cereb. Cortex 21, 1674-1694. doi: 10.1093/cercor/bhq238

Shitamukai, A., Konno, D., and Matsuzaki, F. (2011). Oblique radial glial divisions in the developing mouse neocortex induce self-renewing progenitors outside the germinal zone that resemble primate outer subventricular zone progenitors. J. Neurosci. 31, 3683-3695. doi: 10.1523/JNEUROSCI.477310.2011

Smart, I. H. M., Dehay, C., Giroud, P., Berland, M., and Kennedy, H.
(2002). Unique morphological features of the proliferative zones and postmitotic compartments of the neural epithelium giving rise to striate and extrastriate cortex in the monkey. Cereb. Cortex 12, 37-53. doi: 10.1093/cercor/12. 1.37

Tabata, H., Kanatani, S., and Nakajima, K. (2009). Differences of migratory behavior between direct progeny of apical progenitors and basal progenitors in the developing cerebral cortex. Cereb. Cortex 19, 2092-2105. doi: 10.1093/cercor/ bhn227

Tabata, H., and Nakajima, K. (2001). Efficient in utero gene transfer system to the developing mouse brain using electroporation: visualization of neuronal migration in the developing cortex. Neuroscience 103, 865-872. doi: $10.1016 /$ S0306-4522 (01)00016-1

Tabata, H., and Nakajima, K. (2003). Multipolar migration: the third mode of radial neuronal migration in the developing cerebral cortex. J. Neurosci. 23, 9996-10001.

Tabata, H., Yoshinaga, S., and Nakajima, K. (2012). Cytoarchitecture of mouse and human subventricular zone in developing cerebral neocortex. Exp. Brain Res. 216, 161-168. doi: 10.1007/s00221-011-2933-3

Tachikawa, K., Sasaki, S., Maeda, T., and Nakajima, K. (2008). Identification of molecules preferentially expressed beneath the marginal zone in the developing cerebral cortex. Neurosci. Res. 60, 135-146. doi: 10.1016/j.neures. 2007.10.006

Tárraga, J., Medina, I., Arbiza, L., Huerta-Cepas, J., Gabaldón, T., Dopazo, J., et al. (2007). Phylemon: a suite of web tools for molecular evolution, phylogenetics and phylogenomics. Nucleic Acids Res. 35, W38-W42. doi: 10.1093/nar/ gkm224

Vilella, A. J., Severin, J., UretaVidal, A., Heng, L., Durbin, R., and Birney, E. (2009). EnsemblCompara GeneTrees: 
complete, duplication-aware phylogenetic trees in vertebrates. Genome Res. 19, 327-335. doi: 10.1101/gr. 073585.107

Wang, X., Tsai, J.-W., LaMonica, B., and Kriegstein, A. R. (2011). A new subtype of progenitor cell in the mouse embryonic neocortex. Nat. Neurosci. 14, 555-561. doi: 10.1038/ nn. 2807

Wu, H.-H., Bellmunt, E., Scheib, J. L., Venegas, V., Burkert, C., Reichardt, L. F., et al. (2009). Glial precursors clear sensory neuron corpses during development via Jedi-1, an engulfment receptor. Nat. Neurosci. 12, 1534-1541. doi: 10.1038/nn.2446

Yang, Z. (1998). Likelihood ratio tests for detecting positive selection and application to primate lysozyme evolution. Mol. Biol. Evol. 15, 568-573. doi: 10.1093/ oxfordjournals.molbev.a025957
Yang, Z. (2007). PAML 4: phylogenetic analysis by maximum likelihood. Mol. Biol. Evol. 24, 1586-1591. doi: $10.1093 / \mathrm{molbev} / \mathrm{msm} 088$

Yang, Z., and Nielsen, R. (2002). Codon-substitution models for detecting molecular adaptation at individual sites along specific lineages. Mol. Biol. Evol. 19, 908-917. doi: $10.1093 /$ oxfordjournals. molbev.a004148

Yang, Z., Wong, W. S. W., and Nielsen, R. (2005). Bayes empirical bayes inference of amino acid sites under positive selection. Mol. Biol. Evol. 22, 1107-1118. doi: 10.1093/ molbev/msi097

Zhang, J. (2003). Evolution of the human ASPM gene, a major determinant of brain size. Genetics 165, 2063-2070.

Zhang, J., Nielsen, R., and Yang, Z. (2005). Evaluation of an improved branch-site likelihood method for detecting positive selection at the molecular level. Mol. Biol. Evol. 22, 2472-2479. doi: 10.1093/molbev/ msi237

Zhou, Z., Hartwieg, E., and Horvitz, H. R. (2001). CED-1 is a transmembrane receptor that mediates cell corpse engulfment in C. elegans. Cell 104, 43-56. doi: 10.1016/ S0092-8674(01)00190-8

Conflict of Interest Statement: The authors declare that the research was conducted in the absence of any commercial or financial relationships that could be construed as a potential conflict of interest.

Received: 10 March 2013; paper pending published: 09 April 2013; accepted: 05 July 2013; published online: 31 July 2013.
Citation: Tabata H, Hachiya T, Nagata $K$, Sakakibara $Y$ and Nakajima $K$ (2013) Screening for candidate genes involved in the production of mouse subventricular zone proliferative cells and an estimation of their changes in evolutionary pressure during primate evolution. Front. Neuroanat. 7:24. doi: 10.3389/ fnana.2013.00024

Copyright (C) 2013 Tabata, Hachiya, Nagata, Sakakibara and Nakajima. This is an open-access article distributed under the terms of the Creative Commons Attribution License (CC BY). The use, distribution or reproduction in other forums is permitted, provided the original author(s) or licensor are credited and that the original publication in this journal is cited, in accordance with accepted academic practice. No use, distribution or reproduction is permitted which does not comply with these terms. 